PAPER

\title{
Mapping the onset and progression of atrophy in familial frontotemporal lobar degeneration
}

\author{
J C Janssen, J M Schott, L Cipolotti, N C Fox, R I Scahill, K A Josephs, J M Stevens, M N Rossor
}

J Neurol Neurosurg Psychiatry 2005;76:162-168. doi: 10.1136/jnnp.2003.032201

See end of article for authors' affiliations

\section{Correspondence to:}

Professor M N Rossor, Dementia Research Group, Institute of Neurology, Queen Square, London WCIN 3BG, UK; m.rossor@dementia.ion. ucl.ac.uk

Received

12 November 2003

In revised form

30 April 2004

Accepted 8 May 2004
Background: Frontotemporal lobar degeneration (FTLD) may be inherited as an autosomal dominant disease. Studying patients "at risk" for developing FTLD can provide insights into the earliest onset and evolution of the disease.

Method: We carried out approximately annual clinical, MRI, and neuropsychological assessments on an asymptomatic 51 year old "at risk" family member from a family with FTLD associated with ubiquitinpositive and tau-negative inclusion bodies. We used non-linear (fluid) registration of serial MRI to determine areas undergoing significant regional atrophy at different stages of the disease.

Results: Over the first 26 months of the study, the patient remained asymptomatic, but subsequently developed progressive speech production difficulties, and latterly severe orofacial dyspraxia, dyscalculia, frontal executive impairment, and limb dyspraxia. Regional atrophy was present prior to the onset of symptoms, and was initially centred on the left dorsolateral prefrontal cortex and the left middle frontal gyrus. Latterly, there was increasing asymmetric left frontal and parietal atrophy. Imaging revealed excess and increasing global atrophy throughout the study. Neuropsychological evaluation revealed mild intellectual impairment prior to the onset of these clinical symptoms; frontal executive and left parietal impairment subsequently emerged, culminating in widespread cognitive impairment. Fluid registered MRI allowed the emerging atrophy patterns to be delineated.

Conclusion: We have demonstrated the onset and progressive pattern of in vivo atrophy in familial FTLD using fluid registered MRI and correlated this with the clinical features. Fluid registered MRI may be a useful technique in assessing patterns of focal atrophy in vivo and demonstrating the progression of degenerative diseases.
$\mathrm{F}$ rontotemporal lobar degeneration (FTLD) is a collective term for disorders characterised by focal frontal and temporal lobar atrophy, and clinically by behavioural changes and language deficits. Consensus criteria describe three prototypical clinical FTLD syndromes: frontotemporal dementia (FTD), progressive non-fluent aphasia (PA), and semantic dementia (SD). ${ }^{2}$ FTD is characterised by early personality change and disordered social conduct; PA commences as a disorder of expressive language; and the hallmark of SD is an impairment of comprehension of word meaning and/or object identity. In all three syndromes, perceptual and spatial functions, praxis, and memory are generally intact or relatively well preserved until the disease is advanced. The underlying neuropathological substrate is variable and the clinical manifestations are better related to the topographical distribution of the atrophy rather than to specific histopathological findings.

Approximately $30-50 \%$ of patients with FTLD have a family history of early onset dementia (EOD) with an autosomal dominant pattern of inheritance. ${ }^{2-4}$ The largest group of these families have tau-positive neuronal and glial inclusions at histology, which are usually associated with mutations in the tau gene (MAPT: microtubule associated protein tau) on chromosome $17 \mathrm{q} 21 .^{5-7}$ A further proportion of families with autosomal dominant FTLD have ubiquitin-positive, tau-negative inclusions with or without clinical features of amyotrophic lateral sclerosis. A minority of these ubiquitinpositive and tau-negative families show linkage either to chromosome $9^{8}$ or $17 \mathrm{q} 21 .^{9-12}$ Finally, a proportion of families have no distinctive histopathological findings; a single Danish family in this latter group shows linkage to chromosome $3 .^{13}$
The occurrence of autosomal dominant dementias allows the possibility of gaining unique insights into their pathogenesis and earliest clinical features. By following healthy individuals "at risk" for autosomal dominant disease, it has been possible to demonstrate the clinical, neuropsychological, and imaging changes occurring at the very earliest (presymptomatic) stages of Alzheimer's disease (AD). ${ }^{14}$ The development of techniques such as non-linear (fluid) registration of serial MR images, has allowed the emergence and progression of regional atrophy to be revealed in vivo. ${ }^{15-17}$

We describe the use of fluid registration to determine the progression of atrophy and clinical and neuropsychological features in an initially asymptomatic patient who proceeded to develop FTLD.

\section{METHODS}

Family

This is a British kindred with seven individuals affected with EOD spanning three generations (fig 1). There are few clinical data for earlier generations. Patient III.l died aged 57 years of lobar pneumonia, with a diagnosis of pre-senile dementia. Patient III.2 died at age 60 years of "cerebral sclerosis" in a psychiatric hospital. Patient III.4 died of bronchopneumonia, with an underlying diagnosis of presenile dementia, in the same hospital at age 58 years. No notes survive for any of these family members.

Abbreviations: AD, Alzheimer's disease; BBSI, brain boundary shiff integral; EOD, early onset dementia; FTD, frontotemporal dementia; FTLD, frontotemporal lobar degeneration; MAPT, microtubule associated protein tau; NART, National Adult Reading Test; PA, progressive nonfluent aphasia; TIV, total intracranial volume; SD, semantic dementia; WAIS-R, Wechsler Adult Intelligence Scale-Revised 


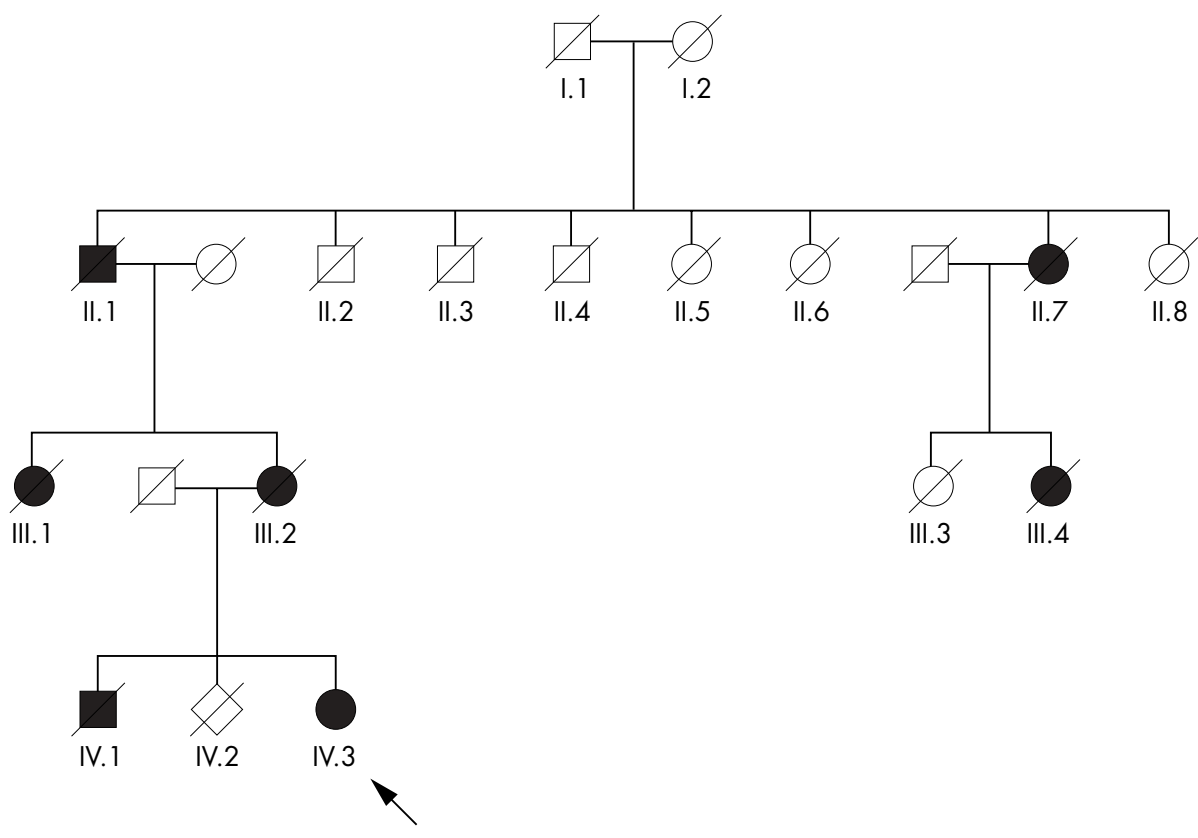

Figure 1 Family tree with index patient marked with arrow. Circles represent females, squares males, and diamonds are anonymised individuals. Filled symbols represent affected family members. Deceased family members are marked with a diagonal slash.
Patient IV.I was assessed when aged 56 years. At that time there was a 4 year history of personality change, disordered social conduct, and cognitive decline. He was wheelchair bound and incontinent, laughed in response to questioning or any attempt to engage him in conversation, and had no comprehensible speech. Brain CT revealed generalised involutional changes particularly prominent over the frontal and temporal areas. He died 12 months later and underwent neuropathological examination. His brain was severely atrophic with the left frontal and temporal lobes most affected. Microscopy revealed no plaques or tangles but showed neuronal loss and astrocytosis with status spongiosus in the superficial layers of the temporal and especially frontal cortex. Immunohistochemistry for ubiquitin revealed a small number of intracytoplasmic inclusions in the hippocampal dentate granule cells, and abnormal neurites and intracytoplasmic inclusions in the superficial layer of the frontal and temporal lobe cortices, as well as intranuclear inclusions in the limbic cortices. Tau and $\alpha$-synuclein immunohistochemistry was negative. The postmortem diagnosis was of familial FTLD with ubiquitin-positive (tau and synuclein negative) inclusions. Although the family was too small for formal linkage studies screening for tau gene mutations was negative.

\section{Prospective study}

The index case, patient IV.3, was enrolled into our prospective longitudinal "at risk" study aged 51 years. Approval was received from the ethics committees of both the National Hospital for Neurology and Neurosurgery and St Mary's Hospital, and the patient gave informed, written consent.

\section{NEUROLOGICAL AND NEUROPSYCHOLOGICAL TESTING}

Assessments were conducted approximately annually and patient IV.3 underwent a standard clinical interview and neurological examination, and was tested on the MiniMental State Examination (MMSE). ${ }^{18}$

The neuropsychological assessment comprised a comprehensive cognitive battery (see also Janssen et $a l^{1920}$ ). The following areas of cognition were investigated: general intellectual functions (shortened version of the Wechsler Adult Intelligence Scale-Revised (WAIS-R), ${ }^{21}$ Advanced Progressive
Matrices $^{22}$ ); verbal recognition and recall memory (verbal version of the Recognition Memory Test, ${ }^{23}$ Paired Associate Learning $\mathrm{Test}^{24}$ ); visual memory (visual version of the Recognition Memory Test, ${ }^{23}$ Topographical Memory Test ${ }^{24}$ ); nominal function (Graded-Difficulty Naming Test ${ }^{25}$ ); word comprehension (Synonyms Test ${ }^{26}$ ); literacy (National Adult Reading Test (NART) ${ }^{27}$ ); spelling (Graded-Difficulty Spelling Test $^{28}$ ); arithmetic (Graded-Difficulty Arithmetic Test ${ }^{29}$ ); visual perceptual and visuospatial functions (Silhouettes and Cubes from the Visual Object and Space Perception Battery $^{30}$ ); frontal "executive" functions (Modified Card Sorting Test, ${ }^{31}$ Cognitive Estimates Test, ${ }^{32}$ Trail Making Test $^{33}$ Hayling Sentence Completion Test, ${ }^{34}$ Weigl Colour Form Sorting Test $^{35}$ ); and speed and attention (Digit Copying and Cancelling $\left.0^{\prime} \mathrm{s}^{36}\right)$.

\section{NEUROIMAGING}

\section{Acquisition and pre-processing}

Seven Tl weighted volumetric MRI scans were acquired over a period of 68 months using two scanners (the first three scans were performed on one scanner, the subsequent four scans on the other). Both scanners were 1.5T GE Signa Units (General Electric, Milwaukee, WI). Scans were acquired using a spoiled gradient echo technique $(256 \times 128 \times 128$ matrix, FOV $24 \times 24 \times 19.2 \mathrm{~cm}$, TR/TE/NEX/ FLIP $=35 / 5 / 1 / 35^{\circ}$ ). This yielded 124 contiguous $1.5 \mathrm{~mm}$ thick slices, which were transferred to a Sun Enterprise 250 workstation (Sun Microsystems, Mountain View, CA) for analysis.

\section{Qualitative assessment and calculation of global atrophy}

Each MRI scan was reported by an experienced neuroradiologist blinded to the clinical information. Image processing was performed using the MIDAS software tool. ${ }^{37}$ A semiautomated technique, using intensity thresholding and a series of erosions and dilations, was applied to delineate brain tissue. Total intracranial volumes (TIV) were measured for each scan.$^{38}$ A rigid body 9 degrees of freedom registration was performed to align the repeat scan onto the baseline image. ${ }^{39}$ Brain volumes for each scan were calculated directly from the MIDAS software tool and corrected for TIV. Whole brain atrophy rates were calculated between intervals from 
the registered scans using a validated automated technique, the brain boundary shift integral (BBSI). ${ }^{40}{ }^{41}$

\section{Assessment of regional atrophy: fluid registration}

The fluid registration model has been described previously. ${ }^{42}{ }^{43}$ Using the rigidly aligned repeat scan as a starting point, the model generates a voxel-level deformation field. The determinant of the Jacobian matrix from the deformation field gives a measure of volume change at the voxel level, and voxel compression maps are created by using a colour overlay to signify this volume change.

\section{RESULTS}

Patient IV.3 attended for nine visits. Neuropsychological assessments were performed at six of these time points, and MRI was performed at seven. Details of these assessments at each time point are presented below, with an overview shown in fig 2. The neuropsychological scores are summarised in table 1 .

\section{Patient IV.3}

At visit 1 patient IV.3 was 51 years old. She was left handed and had been working as a librarian for 26 years. She did not smoke and only rarely drank alcohol. She reported no cognitive or behavioural symptoms, and the clinical assessment was unremarkable, with no evidence of cognitive impairment.

At neuropsychological assessment (1), on the shortened version of the WAIS-R, she scored at an average level on the Verbal scale (VIQ 97) and at the upper end of the low average range on the Performance scale (PIQ 87). She performed at an average level on the Advanced Progressive Matrices. Her premorbid optimal level of functioning was estimated using the NART to be in the superior range (FIQ 123). ${ }^{27}$ This discrepancy indicates a significant intellectual decline. ${ }^{44}$ However, there was no evidence for deficits in memory or visuoperceptual or frontal executive function.

Cross sectional MRI (1) was reported as normal; however, the initial calculated brain volume was slightly small at $1099 \mathrm{ml}$ or $82 \%$ of TIV (normal range: $83-89 \%$ ).

\section{Visit 2 (14 months)}

At the second assessment, she again reported no cognitive or behavioural changes. There was no evidence for decline on neuropsychological testing (2). The MRI (2) was reported as normal.

Applying the automated fluid registration technique to MRI (1) and (2) revealed very focal left frontal atrophy, almost completely confined to the pars opercularis, occurring over the first 14 month period (fig 3).

\section{Visit 3 (26 months)}

At the third assessment the patient remained well. Neuropsychological assessment (3) revealed that her verbal and performance IQs remained static. Despite intact naming skills she performed poorly on a verbal phonemic fluency task. However, she passed three further frontal tasks. Performance on other focal cognitive tasks including memory remained unchanged.

The MRI (3) was reported as normal. Using rigid body registration and the BBSI we demonstrated that over the time period spanning the first three assessments there was $22.9 \mathrm{ml}$ brain volume loss (assuming constant brain loss this equates to $0.96 \%$ of brain volume/year) (normal range: $0.1-0.54 \%$ / year).

Fluid registration was applied to MRIs (2) and (3). Over this 12 month period diffuse left sided frontal lobe atrophy had developed, although the pars opercularis was still losing volume at the fastest rate. There was now clear, but lesser involvement of the pars triangualis, the pars orbitalis, and the middle frontal gyrus. The anterior caudate was slightly involved, but the primary motor cortex, paracentral lobule, temporal lobe structures (including the hippocampi), and cerebellum remained unaffected (figs 4 and 5).

\section{Visit 4 (45 months)}

At the fourth assessment, 19 months later, for the first time the patient reported symptoms. She was having difficulties expressing herself, and felt that her childhood stammer had re-emerged, although this was not apparent during the interview. She reported some difficulties with mental arithmetic. Throughout the interview she appeared more

Table 1 Summary of neuropsychological assessments

\begin{tabular}{|c|c|c|c|c|c|c|}
\hline Assessments & 1 & 2 & 3 & 4 & 5 & 6 \\
\hline Verbal IQ & 97 & 108 & 103 & 97 & 85 & 75 \\
\hline Performance IQ & 87 & 96 & 89 & 92 & 85 & 72 \\
\hline Advanced Matrices & $7 / 12$ & NT & $6 / 12$ & $6 / 12$ & NT & NT \\
\hline NART IQ & 123 & NT & NT & NT & NT & NT \\
\hline RM Words & $>75 \%$ ile & $>75 \%$ ile & $=90 \%$ ile & 75-90\%ile & $=50 \%$ ile & $<5 \%$ ile \\
\hline RM Faces & $>75 \%$ ile & $=75 \%$ ile & $>95 \%$ ile & $=75 \%$ ile & $=25 \%$ ile & $50-75 \%$ ile \\
\hline Topographical & NT & NT & NT & NT & $=95 \%$ ile & $=50 \%$ ile \\
\hline Paired Associates 1 & $>90 \%$ ile & $>90 \%$ ile & $=75 \%$ ile & $=50 \%$ ile & $=75 \%$ ile & $>75 \%$ ile \\
\hline Paired Associates 2 & $>90 \%$ ile & >90\%ile & >90\%ile & $=25 \%$ ile & $>75 \%$ ile & 10-25\%ile \\
\hline Grading Naming & $75-90 \%$ ile & $75-90 \%$ ile & $=95 \%$ ile & $75-90 \%$ ile & $75-90 \%$ ile & $25-50 \%$ ile \\
\hline Synonyms & 75-90\%ile & 75-90\%ile & 75-90\%ile & 50-75\%ile & $=50 \%$ ile & $1-5 \%$ ile \\
\hline GD Spelling & $>90 \%$ ile & $>90 \%$ ile & $>90 \%$ ile & $50-75 \%$ ile & $10-25 \%$ ile & $0 / 30$ \\
\hline GD Arithmetic & $=50 \%$ ile & $25-50 \%$ ile & $25-50 \%$ ile & $10-25 \%$ ile & $=5 \%$ ile & $0 / 24$ \\
\hline Silhouettes & $=50 \%$ ile & $25-50 \%$ ile & $50-75 \%$ ile & $50-75 \%$ ile & $25-50 \%$ ile & $10-25 \%$ ile \\
\hline Cube Analysis & $>5 \%$ cut off & $>5 \%$ cut off & $>5 \%$ cut off & $>5 \%$ cut off & $<5 \%$ cut off & $<5 \%$ cut off \\
\hline WCST & $<50 \%$ cut off & $<50 \%$ cut off & $<50 \%$ cut off & NT & $>50 \%$ cut off & NT \\
\hline Cognitive Estimates & 4 & 3 & 2 & NT & NT & NT \\
\hline "S" words & NT & NT & 9 & 8 & 6 & 1 \\
\hline Trail Making A & NT & NT & 25-50\%ile & 52 (25-50\%ile) & 10-25\%ile & Unable to complete \\
\hline Trail Making B & NT & NT & $25-50 \%$ ile & 120 (25-50\%ile) & $=10 \%$ ile & Failed practice \\
\hline Hayling & NT & NT & NT & 1 (impaired) & 3 (poor) & 3 (poor) \\
\hline Weigl & NT & NT & NT & NT & NT & Pass \\
\hline Digit Copying & $50-75 \%$ ile & $=50 \%$ ile & $50-75 \%$ ile & 41 (50-75\%ile) & $50-75 \%$ ile & $10-25 \%$ ile \\
\hline Cancelling $O^{\prime} s$ & $67(X=46 ; S D=14)$ & $64(X=46 ; S D=14)$ & $48(X=46 ; S D=14)$ & $51(X=46 ; S D=14)$ & $64(X=46 ; S D=14)$ & $51(X=46 ; S D=14)$ \\
\hline
\end{tabular}




\begin{tabular}{|c|c|c|c|c|c|c|c|c|}
\hline Month & 0 & & 26 & 45 & 51 & 56 & 57 & 69 \\
\hline \multirow[t]{3}{*}{ MMSE } & 29 & \multicolumn{2}{|c|}{29} & 29 & 26 & & \multicolumn{2}{|l|}{21} \\
\hline & 1 & \multicolumn{2}{|c|}{2} & 4 & 5 & \multicolumn{2}{|l|}{6} & 7 \\
\hline & \multicolumn{3}{|c|}{ Scans reported normal } & \multicolumn{5}{|c|}{ Increasingly abnormal scans } \\
\hline \multirow[t]{2}{*}{ MRI } & \multicolumn{3}{|c|}{$0.96 \%$ brain volume loss/year } & \multicolumn{3}{|c|}{$4.5 \%$ brain volume loss/year } & \multicolumn{2}{|c|}{$8 \%$ brain volume loss/year } \\
\hline & $\begin{array}{r}\text { Fluid } \\
\text { Ve } \\
\text { fro }\end{array}$ & & $\begin{array}{l}\text { Fluid Registration } 2 \\
\text { Focal, increasing } \\
\text { left frontal atrophy }\end{array}$ & $\begin{array}{r}\text { Flu } \\
\text { Increasing }\end{array}$ & $\begin{array}{l}\text { tratio } \\
\text { ital a } \\
\text { ophy }\end{array}$ & etal & & \\
\hline
\end{tabular}

\begin{tabular}{|c|c|c|c|c|c|c|}
\hline \multirow{4}{*}{ Clinical } & & & & \multicolumn{2}{|c|}{ Increasing speech impairment } & Mute \\
\hline & & & & & \multicolumn{2}{|c|}{ Orofacial and generalised dyspraxia } \\
\hline & & & & & \multicolumn{2}{|l|}{ Primitive reflexes } \\
\hline & \multicolumn{4}{|c|}{ Working } & \multicolumn{2}{|l|}{ Unable to work } \\
\hline N-psych & $\begin{array}{l}\text { Mild } \\
\text { intellectual } \\
\text { impairment }\end{array}$ & $\begin{array}{l}\text { No progressive } \\
\text { intellectual } \\
\text { impairment }\end{array}$ & $\begin{array}{c}\text { Onset of } \\
\text { verbal fluency } \\
\text { deficit }\end{array}$ & $\begin{array}{l}\text { Increasing frontal and left parietal } \\
\text { lobe dysfunction. Memory, } \\
\text { speed, and attention normal }\end{array}$ & No & estable \\
\hline
\end{tabular}

Figure 2 Overview of the clinical, neuropsychological, and MRI assessments that patient IV.3 underwent during the course of the study. N-psych, neuropsychology.

jocular than previously, and laughed inappropriately on occasion.

Neuropsychological testing (4) revealed that her verbal and performance IQs remained unchanged. Her spelling and arithmetic had declined further. Nonetheless, memory, naming, speed and attention, visual perceptual, and visuospatial skills were unchanged. She obtained an impaired score on the Hayling Sentence Completion Test. Despite good scores on tests of speed and attention, her performance on the Trail Making Test was slow.

For the first time her MRI (4) was abnormal, revealing enlargement of the lateral ventricles and widening of the sylvian fissure on the left.

\section{Visit 5 (51 months)}

By the time of the fifth assessment, 6 months later, the patient reported significant deterioration. She now had clear difficulties with expressive language yet was remarkably unconcerned by her symptoms. She had speech production difficulties and made several phonemic errors in both spontaneous speech and when reading a prose passage. She laughed inappropriately on several occasions and manifested bilateral limb dyspraxia. She had orofacial dyspraxia. She failed bedside tests of executive function. Neuropsychological testing (5) revealed a decline in her verbal and performance IQs. Her memory and naming continued to be unaffected, although single word comprehension had declined slightly. Spelling and calculation had declined further. There was impairment of visuospatial function, although visuoperceptual skills remained normal. There was evidence of further frontal executive impairment, with decline on the Wisconsin Card Sorting and Trail Making Tests. Her performance on tests of speed and attention remained normal.
Standard MRI (5) demonstrated further cerebral atrophy, with increasingly prominent lateral ventricles and widening of the left peri-sylvian fissure.

\section{Visit 6 (56 months)}

At the sixth assessment she was seen at the Specialist Cognitive Disorders Clinic. She had previously attended alone, not wanting to burden her husband with taking part in a research project. Her husband was therefore for the first time able to provide a corroborative history. He had been aware of his wife's difficulties expressing herself for about a year. Her childhood stammer had reappeared and her arithmetic had declined. She had become less tidy, but had never got lost. He also reported that she laughed inappropriately and that her food preferences had changed. There was no change in religiosity or libido. Clinical examination was unchanged.

Neuropsychological assessment (6) revealed further intellectual decline. For the first time there was evidence of language and verbal memory impairment. In contrast visual memory remained at a good average level. She failed to score on tests of calculation and spelling. Visuospatial and visuoperceptual skills had declined further. She failed all frontal executive tests, with the exception of the Weigl. Her performance on speed and attention tests remained within normal limits. Subsequently she was no longer able to tolerate formal neuropsychological testing.

The MRI scan (6) was clearly abnormal with prominent ventricles, and asymmetric left temporal lobe atrophy. Atrophy calculated using the BBSI showed that over the 11 month interval between scans (4) and (6), $43.8 \mathrm{ml}$ of brain volume was lost (that is $4.5 \%$ of brain volume/year, assuming linear decline). 
Fluid registration of MRIs (4) and (6) revealed increasing atrophy particularly involving the left orbito-frontal region and right anterior frontal lobe, including the operculum, over the 11 month period. There was more widespread atrophy diffusely involving the left temporal lobe, with the inferior temporal lobe gyrus and, to a lesser extent, the middle temporal gyrus being affected. There was also involvement of the left parietal lobe and both frontal lobes, with relative sparing of the orbito-frontal gyri. The primary sensory and motor cortices, superior temporal gyri, hippocampi, amygdalae, and cerebellum remained unaffected.

\section{Visit 7 (57 months)}

One month later she was admitted for investigation at the National Hospital. Clinical examination revealed her to be perseverative and to exhibit utilisation behaviour and bilateral grasp reflexes; there were no fasciculations and the remainder of the examination was unchanged from her previous assessment. Screening blood tests for reversible causes of dementia were normal or negative. The CSF was acellular, protein and glucose were normal, and oligoclonal bands were absent. The EEG was normal with preservation of $\alpha$ rhythm. She was diagnosed with familial FTLD.

\section{Visit 8 (66 months)}

By this stage the patient was practically mute; she would say "yes" in response to questions, but not always appropriately.

\section{Visit 9 (69 months)}

At this final assessment, the patient's only vocalisation was a giggle. She no longer recognised her friends. Her sense of humour had become puerile and she continued to enjoy simple comedy shows on television. Her oro-facial apraxia had deteriorated and interfered with mastication.

The MRI (7) revealed further asymmetric left temporal atrophy, however, even at this stage frontal lobe atrophy was not prominent. Using the BBSI to compare scans (6) and (7) (an interval of 13 months) $76.3 \mathrm{ml}$ of brain volume was seen to have been lost ( $8 \%$ of brain volume/year).

\section{DISCUSSION}

We report the clinical, neuropsychological, and imaging findings of a patient with familial FTLD over the time period she progressed from being asymptomatic to being profoundly affected. Using fluid registration we demonstrate early left sided focal brain atrophy with subsequent progressive clinical and neuropsychological decline. It seems reasonable to assume that this focal atrophy is responsible for the observed deficits years before the disease would have presented clinically. In addition, the focal left sided atrophy with associated speech production deficits is compelling evidence that our patient, despite being left handed, was left hemisphere dominant.

Cross sectional MRI has been used to demonstrate strikingly different patterns of regional atrophy within the subgroups of FTLD. ${ }^{45}{ }^{46}$ However, demonstrating the progression of atrophy, a hallmark of neurodegeneration, requires serial imaging. Analysis of serial MRI from the same patient can provide an accurate measure of change within an individual over time. Significantly increased rates of global atrophy have been demonstrated in individuals with $\mathrm{AD}$ and FTLD $^{47} 48$ when compared with control subjects. In this study, we found excess global atrophy was occurring from the start of the study and accelerated during the course of the disease in line with both the clinical and neuropsychological progression.

The development of non-linear matching algorithms (fluid registration) allows visualisation of the progression of regional brain atrophy in vivo. This technique has been validated in both $\mathrm{AD}$ and control subjects, ${ }^{42}$ and has been used to demonstrate presymptomatic change in individuals at genetic risk of $\mathrm{AD} .{ }^{15}$ To our knowledge, this is the first study using this technique to report presymptomatic brain atrophy in FTLD.

At this patient's first visit, despite the reported lack of symptoms or abnormal clinical findings, it is notable that neuropsychological testing revealed significant intellectual impairment in the setting of well preserved cognitive skills. Recent studies have shown that the performance on tests of general intelligence, in particular tests of fluid intelligence such as the Advanced Matrices, is related to brain function in the lateral prefrontal cortex. ${ }^{49}$ Thus our patient's impaired performance on tests of general intelligence may suggest early frontal dysfunction. However, Geschwind et al have shown that frontal executive impairment may be present in patients destined to develop FTLD decades before the onset of symptoms. ${ }^{50}$ In our patient there was unequivocal evidence of further neuropsychological decline only at the third assessment. This is compatible with the view that the intellectual impairment documented at first assessment may have been longstanding. However, the absence of a practice effect on the patient's performance on the first three administrations of the WAIS-R also raises the possibility that some further intellectual decline was occurring.

Fluid registration of the first and second MRI scans revealed highly selective left sided frontal atrophy almost completely confined to the pars opercularis. This change was accompanied by neither clinical nor neuropsychological evidence of decline. By the third scan this atrophy pattern had progressed and extended to involve the pars triangualis, the pars orbitalis, and, to a lesser extent, the middle frontal gyrus. This increased, but still focal, atrophy was not accompanied by clinical evidence of decline. However, neuropsychological testing did reveal evidence of a new selective verbal (phonemic) fluency deficit, implicating dysfunction in the left dorsolateral prefrontal cortex. A recent review of the literature has proposed that lesions of the dominant pars opercularis may impair the ability to sequence words syntactically, and that lesions of the pars triangualis may impair the ability to generate words. ${ }^{51}$ Positron emission tomography studies have also shown activation of left dorsolateral prefrontal cortex, Broca's area, and anterior cingulate during word generation tasks (for a review see Nathaniel-James and Frith ${ }^{52}$ ). We conclude that the demonstrated atrophy pattern is likely to be responsible for the observed impairment in verbal fluency, but that there was evidence of very focal atrophy in this region that preceded any clinical or neuropsychological evidence of progressive impairment.

Our findings are broadly in keeping with previous functional neuroimaging studies of speech production in normal volunteers ${ }^{53}$ and after stroke. ${ }^{54}$ However, a recent PET study in PA, although demonstrating decreased activation in the caudal part of Broca's area, showed maximum focal cerebral hypometabolism centred around the left anterior insula..$^{55}$ It is notable that all patients in this latter study had established PA; it is therefore possible that insula involvement is a later feature of the disease. Alternatively, the differences may be due to the differences in age, disease duration, or possibly the underlying neuropathophysiology of the patient group. Thus, whilst these studies are clearly complementary, they are not directly comparable.

The subsequent course of this patient's disease was characterised clinically by a progressive decline in speech production and the emergence of increasing frontal executive and dominant parietal dysfunction. By the final neuropsychological assessment (6), there was further significant deterioration of intellectual function, with by now marked 


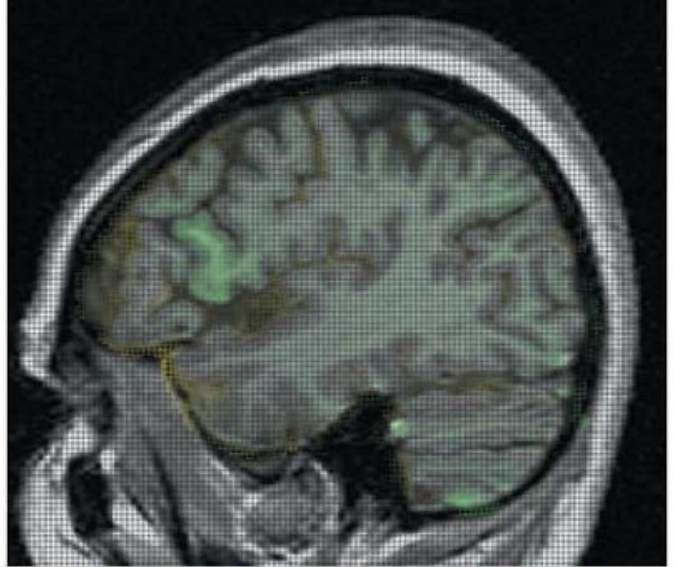

Contracting Expanding

Figure 3 Sagittal MRI with voxel-compression-mapping overlay over time period one, showing very focal anterolateral left frontal lobe atrophy, particularly centred around the pars opercularis.

global impairment of executive function and severe dyscalculia and dysgraphia. Speech production difficulties were by now prominent. At this stage, performance on a demanding series of memory tests remained broadly within normal limits. Similarly, naming, verbal comprehension, and tests of speed and attention were satisfactory.

Fluid registration over the time period of scans (4)-(6) revealed increasing atrophy centred around the left frontal and left parietal lobes, findings consistent with those from clinical assessment and neuropsychological testing. No significant atrophy was seen in the hippocampi and medial temporal lobes, compatible with the patient's well preserved memory. The re-emergence of primitive reflexes, generally implicated in frontal lobe disease, in the absence of long-tract or cerebellar signs is also compatible with the observed atrophy pattern. The increasing rates of global brain atrophy over the period of follow up are in keeping both with the increasing regional atrophy demonstrated using fluid registration, and clinical disease progression. Importantly, there were no abnormalities visible on cross sectional imaging until scan (4), 45 months into the

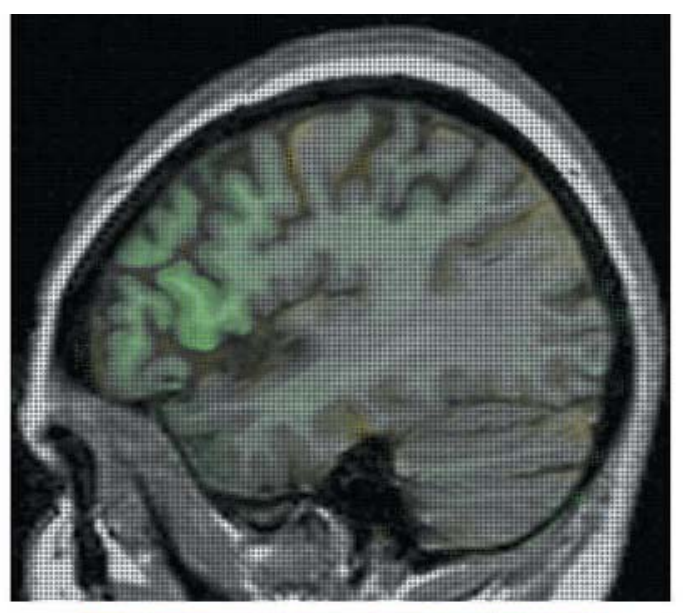

Contracting Expanding

Figure 4 Sagittal MRI with voxel-compression-mapping overlay over time period two, showing focal anterolateral left frontal lobe atrophy, extending from, but still centred around, the pars opercularis.

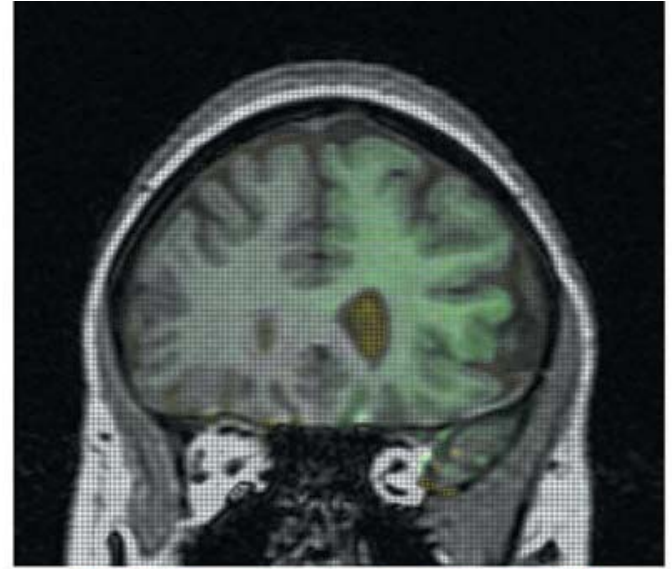

Contracting

Expanding

Figure 5 Coronal MRI with voxel-compression-mapping overlay over time period two, showing marked asymmetric (left $>$ right) frontal lobe atrophy at this stage.

study, and 31 months after clear focal changes had been demonstrated using fluid registration. When abnormalities did become present on conventional imaging, they were entirely in keeping with a diagnosis of FTLD.

This study has a number of limitations. The patient attended alone until the sixth assessment, and the history given may therefore be incomplete. However, when she was accompanied by her husband, his account of her illness was broadly in keeping with our clinical assessment. We have no information concerning the early course of the disease in other affected family members. This patient's brother was not assessed until late in his disease, at which time many of the reported clinical features appear to be consistent with FTLD. Given this, the family history, and similar young age at onset, it is highly likely that both siblings were suffering from the same disease process. However, as FTLD is a clinically and neuropathologically diverse entity, the pattern and progression of atrophy we describe in this individual are unlikely to be consistent amongst the different clinical presentations. Although the use of two different scanners could result in a systematic bias regarding the reported progression of atrophy, the earliest changes were all demonstrated on scans acquired on the first scanner and no "cross scanner" calculations or analyses were performed. Finally, the neuropsychological battery used in this study, although comprehensive, was initially designed to detect the earliest changes in familial AD, and not FTLD per se. Accordingly the selected tests were not specifically targeted to detect early speech production deficits. It is therefore possible that a more detailed assessment may have detected subtle speech production dysfunction even earlier than was documented in our study.

Fluid registered MRI is an unbiased, non-invasive technique that can be used to accurately delineate focal atrophy occurring between serially acquired MRI scans. As this case demonstrates, this methodology may be a useful means of demonstrating the earliest features and progression of degenerative diseases in vivo.

\section{ACKNOWLEDGEMENTS}

We thank the family members and their attendant physicians, the MRI staff at St Mary's Hospital, London; David MacManus at the NMR Research Unit at the Institute of Neurology, London; Jennifer Whitwell for assisting with image analysis; and Dr Tamas Revesz for advice on the histopathology. 


\section{Authors' affiliations}

J C Janssen, J M Schott, N C Fox, R I Scahill, K A Josephs, M N Rossor, Dementia Research Group, Institute of Neurology, London, UK L Cipolotti, Department of Neuropsychology, National Hospital for Neurology and Neurosurgery, London, UK

J M Stevens, Department of Clinical Neuroradiology, National Hospital for Neurology and Neurosurgery, London, UK

J M Stevens, M N Rossor, Division of Neuroscience and Psychological Medicine, Faculty of Medicine, Imperial College of Science, Engineering and Medicine, London, UK

This study was funded by a UK Medical Research Council Program grant G9626876; RIS is supported by the Alzheimer's Research Trust and latterly by the Medical Research Council; JMS is in receipt of an Alzheimer's Society Research Fellowship; and NCF is a Medical Research Council Senior Clinical Scientist.

Competing interests: none declared

Martin Rossor assumed editorship of the Journal of Neurology, Neurosurgery and Psychiatry after submission of this paper and has had no involvement in the review process.

\section{REFERENCES}

1 Neary D, Snowden JS, Gustafson L, et al. Frontotemporal lobar degeneration: a consensus on clinical diagnostic criteria. Neurology 1998;51(6):1546-54

2 McKhann G, Albert MS, Grossman M, et al. Clinical and pathological diagnosis of frontotemporal dementia: report of the Work Group on Frontotemporal Dementia and Pick's Disease. Arch Neurol 2001;58(11):1803-9.

3 Stevens $M$, van Duijn $C M$, Kamphorst $W$, et al. Familial aggregation in frontotemporal dementia. Neurology 1998;50(6): 1541-5.

4 Foster NL, Wilhelmsen $\mathrm{K}$, Sima AA, et al. Frontotemporal dementia and parkinsonism linked to chromosome 17: a consensus conference. Ann Neurol 1997;41(6):706-15.

5 Hutton M, Lendon CL, Rizzu P, et al. Association of missense and 5'-splice-site mutations in tau with the inherited dementia FTDP-17. Nature 1998:393(6686):702-5.

6 Poorkaj P, Bird TD, Wiisman E, et al. Tau is a candidate gene for chromosome 17 frontotemporal dementia. Ann Neurol 1998;43(6):815-25.

7 Spillantini MG, Murrell JR, Goedert M, et al. Mutation in the tau gene in familial multiple system tauopathy with presenile dementia. Proc Natl Acad Sci U S A 1998;95(13):7737-41.

8 Hosler BA, Siddique T, Sapp PC, et al. Linkage of familial amyotrophic lateral sclerosis with frontotemporal dementia to chromosome 9q21-q22. JAMA 2000;284(13): 1664-9.

9 Bird TD, Wiisman EM, Nochlin D, et al. Chromosome 17 and hereditary dementia: linkage studies in three non-Alzheimer families and kindreds with late-onset FAD. Neurology 1997;48(4):949-54.

10 Basun H, Almkvist O, Axelman K, et al. Clinical characteristics of a chromosome 17-linked rapidly progressive familial frontotemporal dementia. Arch Neurol 1997:54(5):539-44.

11 Lendon CL, Lynch T, Norton J, et al. Hereditary dysphasic disinhibition dementia: a frontotemporal dementia linked to 17q21-22. Neurology 1998;50(6): 1546-55.

12 Rosso SM, Kamphorst W, de Graaf B, et al. Familial frontotemporal dementia with ubiquitin-positive inclusions is linked to chromosome 17q21-22. Brain $2001 ; 124(10): 1948-57$

13 Ashworth A, Lloyd S, Brown J, et al. Molecular genetic characterisation of frontotemporal dementia on chromosome 3. Dement Geriatr Cogn Disord 1999:10(suppl 1):93-101.

14 Fox NC, Warrington EK, Seiffer AS, et al. Presymptomatic cognitive deficits in individuals at risk of familial Alzheimer's disease. A longitudinal prospective study. Brain 1998;121:1631-9.

15 Fox NC, Crum WR, Scahill Rl, et al. Imaging of onset and progression of Alzheimer's disease with voxel-compression mapping of serial magnetic resonance images. Lancet 2001;358(9277):201-5.

16 Schott JM, Simon JE, Fox NC, et al. Delineating the sites and progression of in vivo atrophy in multiple system atrophy using fluid-registered MRI. Mov Disord 2003;18(8):955-8.

17 Scahill RI, Schott JM, Stevens JM, et al. Mapping the evolution of regional atrophy in Alzheimer's disease: unbiased analysis of fluid-registered serial MRI. Proc Natl Acad Sci U S A 2002;99(7):4703-7.

18 Folstein M, Folstein S, McHughs P. The "Mini Mental State": a practical method for grading the cognitive state of patients for the clinician. J Psychiatr Res 1975; 12:189-98.

19 Janssen JC, Hall M, Fox NC, et al. Alzheimer's disease due to an intronic presenilin-1 (PSEN1 intron 4) mutation: a clinicopathological study. Brain 2000;123(5):894-907.

20 Janssen JC, Lantos PL, Fox NC, et al. Autopsy-confirmed familial early-onset Alzheimer disease caused by the L153V presenilin 1 mutation. Arch Neurol $2001 ; 58(6): 953-8$.
21 Wechsler D. Manual for the Wechsler Adult Intelligence Scale, Revised. New York: Psychological Corporation, 1981.

22 Raven JC. Advanced Progressive Matrices, sets I and II. London: HK Lewis, 1965.

23 Warrington EK. Manual for the Recognition Memory Test for words and faces. Windsor, UK: NFER-Nelson, 1984.

24 Warrington EK. The Camden Memory Tests manual. Hove: Psychology Press, 1996.

25 McKenna P, Warrington EK. Testing for nominal dysphasia. J Neurol Neurosurg Psychiatry 1980;43(9):781-8.

26 Warrington EK, McKenna P, Orpwood L. Single word comprehension: a concrete and abstract word synonym test. Neuropsychol Rehabil 1998;8:143-54.

27 Nelson HE, Willison J. The National Adult Reading Test (NART) manual, 2nd ed. Windsor, UK: NFER-Nelson, 1991.

28 Baxter DM, Warrington EK. Measuring dysgraphia: a graded-difficulty spelling test. Behar Neurol 1994;7(3-4):107-16.

29 Jackson M, Warrington EK. Arithmetic skills in patients with unilateral cerebral lesions. Cortex 1986;22(4):61 1-20.

30 Warrington EK, James $M$. The visual object and space perception battery. Bury St Edmunds, UK: Thames Valley Test Company, 1991

31 Nelson HE. A modified card sorting test sensitive to frontal lobe defects. Cortex 1976;12:313-24.

32 Shallice T, Evans ME. The involvement of the frontal lobes in cognitive estimation. Cortex 1978;14(2):294-303.

33 Reitan RM. The validity of the trail making test as an indicator of organic brain damage. Percept Mot Skills 1958;8:271-6.

34 Burgess PW, Shallice T. The Hayling Sentence Completion test. Neuropsychologia 1996;34:263-72.

35 Weigl E. On the psychology of the so called process of abstraction. J Abnorm Soc Psychol 1948;36:3-33.

36 Willison JR, Warrington EK. Cognitive retardation in a patient with preservation of psychomotor speed. Behav Neurol 1992:5:113-6.

37 Freeborough PA, Fox NC, Kitney RI. Interactive algorithms for the segmentation and quantitation of 3-D MRI brain scans. Comput Methods Programs Biomed 1997:53(1):15-25.

38 Whitwell JL, Crum WR, Watt HC, et al. Normalization of cerebral volumes by use of intracranial volume: implications for longitudinal quantitative MR imaging. Am J Neuroradiol 2001; 22(8): 1483-9.

39 Freeborough PA, Woods RP, Fox NC. Accurate registration of serial 3D MR brain images and its application to visualizing change in neurodegenerative disorders. J Comput Assist Tomogr 1996;20(6):1012-22.

40 Fox NC, Freeborough PA. Brain atrophy progression measured from registered serial MRI: validation and application to Alzheimer's disease. $J$ Magn Reson Imaging 1997;7(6): 1069-75.

41 Freeborough PA, Fox NC. The boundary shift integral: an accurate and robust measure of cerebral volume changes from registered repeat MRI. IEEE Trans Med Imaging 1997; 16(5):623-9.

42 Freeborough PA, Fox NC. Modeling brain deformations in Alzheimer disease by fluid registration of serial 3D MR images. J Comput Assist Tomogr 1998;22(5):838-43.

43 Crum WR, Scahill RI, Fox NC. Automated hippocampal segmentation by regional fluid registration of serial MRl: validation and application in Alzheimer's disease. Neuroimage 2001;13(5):847-55.

44 Nelson HE, O'Connell A. Dementia: the estimation of premorbid intelligence levels using the New Adult Reading Test. Cortex 1978:14(2):234-44.

45 Chan D, Fox NC, Scahill Rl, et al. Patterns of temporal lobe atrophy in semantic dementia and Alzheimer's disease. Ann Neurol $2001 ; 49(4): 433-42$

46 Perry RJ, Hodges JR. Differentiating frontal and temporal variant frontotemporal dementia from Alzheimer's disease. Neurology 2000;54(12):2277-84.

47 Fox NC, Freeborough PA, Rossor MN. Visualisation and quantification of atrophy in Alzheimer's disease. Lancet 1996;348(9020):94-7.

48 Chan D, Fox NC, Jenkins R, et al. Rates of global and regional cerebral atrophy in $A D$ and frontotemporal dementia. Neurology 2001;57(10): 1756-63.

49 Gray JR, Chabris CF, Braver TS. Neural mechanisms of general fluid intelligence. Nat Neurosci 2003;6(3):316-22.

50 Geschwind DH, Robidoux J, Alarcon M, et al. Dementia and neurodevelopmental predisposition: cognitive dysfunction in presymptomatic subjects precedes dementia by decades in frontotemporal dementia. Ann Neurol 2001;50(6):741-6.

51 Taubner RW, Raymer AM, Heilman KM. Frontal-opercular aphasia. Brain Lang 1999;70(2):240-61

52 Nathaniel-James D, Frith C. The role of the dorsolateral prefrontal cortex evidence from the effects of contextual constraint in a sentence completion task. Neuroimage 2002;16(4):1094.

53 Blank SC, Scott SK, Murphy K, et al. Speech production: Wernicke, Broca and beyond. Brain 2002;125(Pt 8): 1829-38.

54 Blank SC, Bird H, Turkheimer F, et al. Speech production after stroke: the role of the right pars opercularis. Ann Neurol 2003;54(3):310-20.

55 Nestor PJ, Graham NL, Fryer TD, et al. Progressive non-fluent aphasia is associated with hypometabolism centred on the left anterior insula. Brain 2003;126(Pt 11):2406-18. 